Revista Brasileira de Agricultura Irrigada v.12, nº.5, p. 2964 - 2975, 2018

ISSN 1982-7679 (On-line)

Fortaleza, CE, INOVAGRI - http://www.inovagri.org.br

DOI: $10.7127 /$ rbai.v12n500936

Protocolo 936.18 - 23/04/2018 Aprovado em 11/09/2018

\title{
EVAPOTRANSPIRAÇÃO DE REFERÊNCIA PELAS EQUAÇÕES DE HARGREAVES NA MESORREGIÃO DO TRIÂNGULO MINEIRO E ALTO PARANAÍBA - MG
}

\author{
José Eduardo Pitelli Turco ${ }^{1}$, Lucas Hordones Chaves ${ }^{2}$
}

\begin{abstract}
RESUMO
A FAO (Food and Agriculture Organization of the United Nations) recomenda utilizar a equação de Penman-Monteith para estimar a evapotranspiração de referência (ETo), que exige dados meteorológicos que dificultam sua aplicação. Com este trabalho, objetivou-se verificar a eficiência de três equações de Hargreaves comparando-as com a equação de Penman-Monteith, nas condições climáticas da mesorregião do Triângulo Mineiro e Alto Paranaíba - MG, e fazer a calibração local e regional das equações, baseadas na temperatura do ar. Para tanto, utilizouse dados médios diários dos anos de 2009 a 2015, de radiação solar global, velocidade do vento, temperatura do ar e umidade relativa do ar, obtidos em estações meteorológicas automáticas. A análise dos resultados foi realizada por meio da aplicação de técnicas que verificaram a integridade dos dados meteorológicos e para avaliação da estimativa da ETo, foram adotadas metodologias de análise dos dados médios e de desempenho das estimativas. As equações de Hargreaves ajustadas, local e regional, são uma alternativa eficiente para estimar a ETo diária em locais do Triângulo Mineiro e Alto Paranaíba.
\end{abstract}

Palavras-chave: Penman-Monteith, expoente empírico HE, temperatura do ar

\section{REFERENCE EVAPOTRANSPIRATION BY THE HARGREAVES EQUATIONS IN MESOREGION THE TRIÂNGULO MINEIRO AND ALTO PARANAÍBA - MG}

\begin{abstract}
The FAO (Food and Agriculture Organization of the United Nations) recommends the PenmanMonteith equation to estimate reference evapotranspiration (ETo). This work aimed to verify the efficiency of three Hargreaves equations comparing them with the Penman-Monteith, in the climatic conditions of the Triangulo Mineiro and Alto Paranaíba - MG mesoregion, as well as to make the local and regional calibration of the equations based on the air temperature. To this end, we used daily data of the years 2009 to 2015 of global solar radiation, wind speed, air

\footnotetext{
${ }^{1}$ Doutor em Engenharia, Prof. Adjunto III da FCAV/UNESP, Câmpus de Jaboticabal, email: jepturco@fcav.unesp.br

${ }^{2}$ Doutor em Agronomia, Prof. do Instituto Federal do Pará, Campus Cametá, e-mail: lucas.chaves@ifpa.edu.br
} 
temperature and relative humidity of the air obtained in automatic meteorological stations were used. The analysis of the results was performed through the application of techniques that verified the integrity of the meteorological data and to estimate the ETo, here adopted methodologies for the analysis of the average data and the performance of the ETo estimates. The Hargreaves equations are an efficient alter native to estimate the daily values of ETo in the Triangulo Mineiro and Alto Paranaíba mesoregion, places where the availability of climatic data is limited.

Keywords: Penman-Monteith, HE empirical exponent, air temperature

\section{INTRODUÇÃO}

Na mesorregião do Triângulo Mineiro e Alto Paranaíba - MG existe o Comitê de Bacia Hidrográfica do Rio Araguari, pelo qual se instituiu aos usuários a cobrança pelo uso do recurso hídrico da Bacia, a primeira iniciativa no País. Devido a esta cobrança, é de suma importância irrigação adequada para os locais citados.

A irrigação adequada tem por base a estimativa diária da evapotranspiração de referência (ETo), sendo um parâmetro agrometeorológico indispensável para o planejamento e o manejo de irrigação.

O método de estimativa da evapotranspiração recomendado pelo boletim da FAO56 é Penman-Monteith (PM). Entretanto, requer um conjunto amplo de dados que impõe obstáculos à sua difusão. Portanto, a busca por métodos alternativos que demandem menos quantidade de variáveis meteorológicas para estimar a ETo têm sido uma solução viável para contornar esse problema (FERNANDES et al., 2012).

Antes de aplicar um método de estimativa da evapotranspiração de referência para determinado local, é necessário verificar o desempenho deste método e, quando necessário, fazer calibrações a fim de minimizar erros de estimativa. Esse desempenho tem sido analisado com a comparação dos métodos em estudo ao método de Penman-Monteith FAO-56 (PILAU et al., 2012).

Os métodos baseados na temperatura do ar têm sido frequentemente utilizados ou recomendados, conforme destacado por Moura et al., (2013). Um método alternativo que vem sendo utilizado por vários estudos (RAZIEI;
PEREIRA, 2013; HEYDARI et al., 2013; MEHDIZADEH et al., 2016) é a equação de Hargreaves (HARGREAVES; SAMANI, 1985).

Entre os métodos que podem ser usados com apenas dados de temperatura, destacam-se o de Hargreaves (1994). Tal método pode apresentar correções locais de caráter diário, semanal ou mensal que podem afetar a precisão da estimativa do método. Existem estudos que mostram que essa equação ajustada pode estimar com acurácia a ETo diária (BERTI et al., 2014; FENG et al., 2017).

O método de Hargreaves superestima a $\mathrm{ET}_{0}$ em locais de clima úmido, conforme observado por Cervantes-Osornio et al. (2013).

Objetivou-se verificar a eficiência de três equações de Hargreaves comparando-as com a equação de Penman-Monteith, nas condições climáticas da mesorregião do Triângulo Mineiro e Alto Paranaíba - MG, bem como fazer a calibração local e regional das equações, baseadas na temperatura do ar, por meio do ajuste do parâmetro empírico HE (expoente empírico de Hargreaves).

\section{MATERIAIS E MÉTODOS}

A área de estudo (Figura 1) está localizada na mesorregião do Triângulo Mineiro e Alto Paranaíba no estado de Minas Gerais, predominantemente no Bioma Cerrado, composta por serras distribuídas paralelamente aos rios Paranaíba e Grande.

Segundo a classificação de Köeppen, o clima da região é predominante Aw, tropical 
úmido de savana com a época seca coincidindo com o inverno, vegetação arbustiva e rasteira.

Os dados foram obtidos de sete (07) estações meteorológicas automáticas (modelo MAWS301, Vaisala) do Instituto Nacional de
Meteorologia - INMET, situadas na mesorregião do Triângulo Mineiro e Alto Paranaíba - MG, Figura 1, referentes ao período de $1^{\circ}$ de janeiro de 2009 a 31 de dezembro de 2015.

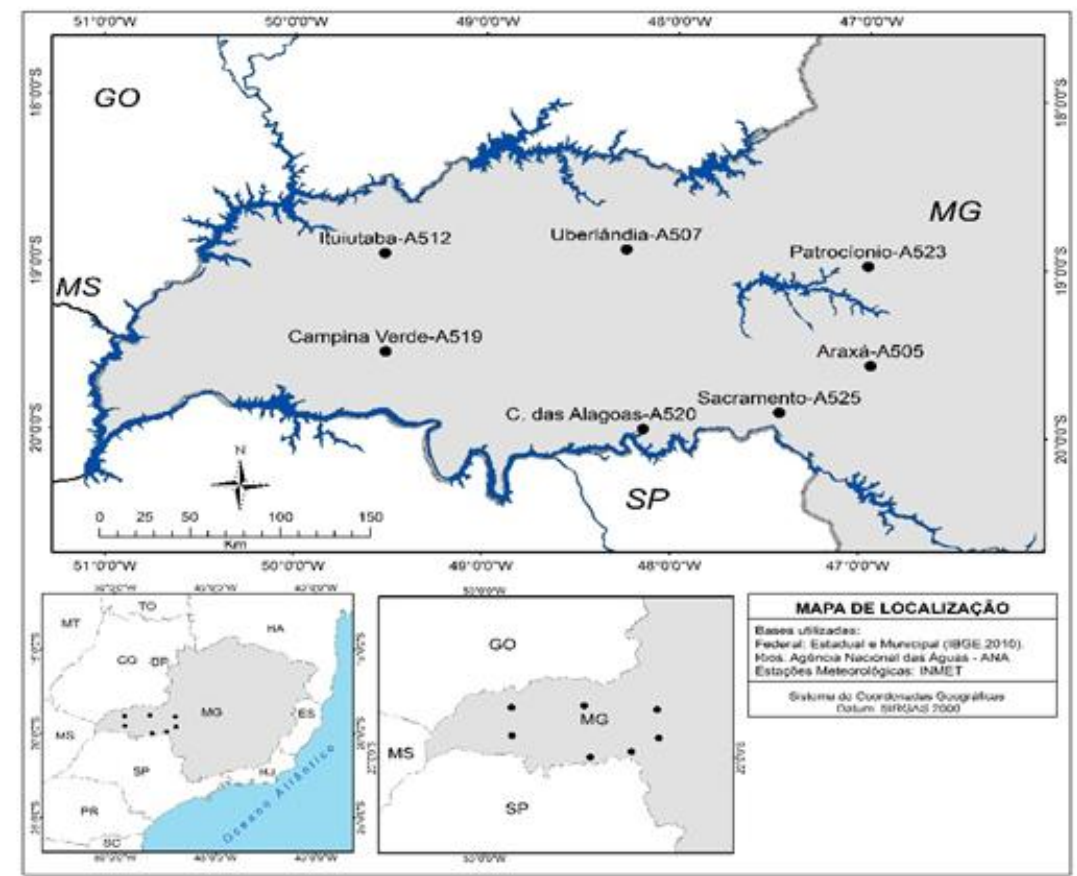

Figura 1. Mapa com a localização geográfica das estações meteorológicas utilizadas no estudo.

A integridade dos dados das estações meteorológicas automáticas foi avaliada pelas técnicas descritas por Allen (1996). Os registros produzidos por estações meteorológicas automáticas foram comparados ao dos postos meteorológicos da mesorregião, que são situados nas cidades de Araxá, Uberaba, Capinópolis e Patos de Minas.

A ETo foi estimada em escala média diária, para os anos de 2009 a 2015, pelas equações de Hargreaves (1976), Hargreaves e Samani (1985), Hargreaves (1994) e PenmanMonteith FAO56 (ALLEN et al., 2006), considerando esse último como padrão para a comparação dos demais métodos.

Equação de Hargreaves (1976)

Seja,

$$
\mathrm{EToH}=0,0075 \mathrm{Q} \mathrm{T}
$$

A equação básica proposta por Hargreaves (1976), para o cálculo da evapotranspiração potencial ou de referência, na qual:

EToH - evapotranspiração de referência, em gramado, $\mathrm{mm} \mathrm{dia}^{-1}$;

$\mathrm{T}$ - temperatura média do ar, ${ }^{\circ} \mathrm{F}$;

$\mathrm{Q}$ - radiação solar medida, cal $\mathrm{cm}^{-2} \mathrm{dia}^{-1}$.

Pode-se escrever a eq. (1) na seguinte forma:

$$
E T o H=0,0075 \frac{Q}{59} T
$$

Em que,

Q - radiação solar global recebida, $\mathrm{cal} \mathrm{cm}^{-2} \mathrm{dia}^{-1}$;

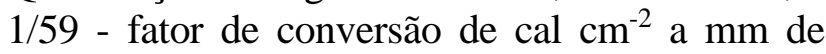
evaporação equivalente.

Equação de Hargreaves e Samani (1985)

$$
\text { Considerando, EToHS }=0,0023 \text { Qo }(\text { Tmáx }- \text { Tmín })^{\mathrm{HE}}(\mathrm{T}+17,8)
$$


Em que,

EToHS - evapotranspiração de referência, grama, $\mathrm{mm} \mathrm{d}^{-1}$;

Tmáx - temperatura máxima, ${ }^{\circ} \mathrm{C}$;

Tmín - temperatura mínima, ${ }^{\circ} \mathrm{C}$;
$\mathrm{T}$ - temperatura média diária, ${ }^{\circ} \mathrm{C}$;

Qo - radiação solar extraterrestre $\left(\mathrm{mm} \mathrm{dia}^{-1}\right)$;

HE - expoente empírico $(0,5)$.

Equação de Hargreaves (1994)

$$
\text { Considerando, EToHG }=0,0023 \mathrm{RA}\left(\mathrm{T}^{\circ} \mathrm{C}+17,8\right) \mathrm{TD}^{\mathrm{HE}}
$$

Em que,

EToHG - evapotranspiração de referência, em gramado, mm dia ${ }^{-1}$;

RA - radiação solar extraterrestre $\left(\mathrm{mm} \mathrm{dia}^{-1}\right)$;

$\mathrm{T}^{\circ} \mathrm{C}$ - (Tmax + Tmin)/2 (temperatura máxima diária do ar mais a temperatura mínima diária do ar, em ${ }^{\circ} \mathrm{C}$, dividido por dois);

TD - Tmax - Tmin (temperatura máxima diária do ar menos a temperatura mínima diária do ar, em ${ }^{\circ} \mathrm{C}$ );

HE - expoente empírico $(0,5)$.
Os valores diários da radiação solar extraterrestre para equação Hargreaves e Samani (1985) e Hargreaves (1994) foram obtidos em função da latitude local, a partir dos dados interpolados de Napier Shaw, citado por Ramos et al. (1989), tendo como parâmetro máximo da atmosfera, o $15^{\circ}$ dia de cada mês entre as latitudes $10^{\circ} \mathrm{N}$ e $40^{\circ} \mathrm{S}$.

Equação do Penman-Monteith (PM)

Para cálculo da evapotranspiração de referência Allen et al. (2006) propuseram:

$$
E T o P M=\frac{0,409 \Delta(R n-G)+\gamma\left(\frac{900}{T+273}\right) v\left(e_{s}-e\right)}{\Delta+\gamma\left(1+0,34_{v}\right)}
$$

Em que,

ETo_PM - evapotranspiração de referência, em gramado, $\mathrm{mm} \mathrm{d}^{-1}$;

$\mathrm{Rn}$ - radiação líquida, $\mathrm{MJ} \mathrm{m}^{-2} \mathrm{~d}^{-1}$;

$\mathrm{G}$ - fluxo de calor no solo, $\mathrm{MJ} \mathrm{m}^{-2} \mathrm{~d}^{-1}$;

$\mathrm{T}$ - temperatura média do ar, ${ }^{\circ} \mathrm{C}$;

$\mathrm{V}$ - velocidade média do vento a $2 \mathrm{~m}$ de altura, $\mathrm{m}$

$\mathrm{s}^{-1}$;

$\left(\mathrm{e}_{\mathrm{s}}\right.$ - e) - déficit de pressão de vapor, $\mathrm{kPa}$;

$\Delta$ - curva de pressão de vapor, $\mathrm{kPa}^{\circ} \mathrm{C}^{-1}$;

$\gamma$ - constante psicrométrica, $\mathrm{kPa}^{\circ} \mathrm{C}^{-1}$;

900 - fator de conversão.

Foi realizada a calibração local (município da estação) das equações de Hargreaves (EToHSL e EToHGL ) e a regional (EToHSR e EToHGR) para mesorregião por meio do ajuste do expoente empírico HE das equações.

Para a calibração das equações, foi utilizando os dados meteorológicos dos anos ímpares da série (2009, 2011, 2013 e 2015). Para validação da equação foram empregados os dados dos anos pares da série (2010, 2012, 2014).
O ajuste do parâmetro da equação de Hargreaves e Samani (1985) e Hargreaves (1994) foi realizado a partir do Microsoft Excel ${ }^{\circledR}$ utilizando a metodologia descrita por Wraith e Or (1998) para ajuste de equação não linear usando o aplicativo Solver. Essa técnica utiliza como parâmetro de otimização a minimização da soma do desvio ao quadrado (equação 6), ou seja, a diferença entre os valores obtidos por PM e por HG em uma calibração perfeita será próxima a zero.

$$
\sum_{i=1}^{n}\left(P M_{i}-H G_{i}\right)^{2}=0
$$

Em que:

PMi - evapotranspiração de referência estimada pelo método de Penman-Monteith (mm dia ${ }^{-1}$ ) para um respectivo dia de um determinado ano (i);

HGi - evapotranspiração de referência estimada pelo método de local ou regional $\left(\mathrm{mm} \mathrm{dia}^{-1}\right)$ para um respectivo dia de um determinado ano; $\mathrm{N}$ - número total de dias. 
Para a análise dos dados médios diários da ETo, antes e após a calibração, os métodos avaliados foram classificados em relação aos seus respectivos desempenhos, a partir do desvio médio absoluto (DMA), desvio médio relativo (DMR), índice de concordância ou índice de Willmott (d), coeficiente de confiança (c), coeficiente de correlação (r) e de determinação $\left(\mathrm{R}^{2}\right)$, adotados por Souza (2011).

O coeficiente c, proposto por Camargo e Sentelhas (1997), é interpretado por classificação de acordo com os valores: "Ótimo" $>0,85$; 0,76< "Muito Bom" >0,85; 0,66 < “Bom” < 0,75; 0,61 < "Mediano'<0,65; "Ruim” $<0,60$.

Para quantificar o desempenho das equações EToHS e EToHG, ajustadas para o processo local e regional, utilizou-se os seguintes critérios estatísticos, o coeficiente de determinação $\left(\mathrm{R}^{2}\right)$, o erro relativo do quadrado médio (ERQM), o erro médio absoluto (EMA). Estes critérios estatísticos são descritos por Fernandes (2012).

Um modelo perfeito deve ter a EF (eficiência do modelo) igual a 1. Um valor igual a 0 significa que o modelo não prediz melhor que a média aritmética dos valores observados, ou seja, o modelo não serve para explicar a variabilidade nos valores observados.

\section{RESULTADOS E DISCUSSÃO}

Adotando os procedimentos descritos por Allen (1996) a integridade dos dados de radiação solar global, temperatura do ar, umidade do ar, velocidade do vento e precipitação pluviométrica, das 7 estações meteorológicas automáticas, foi considerada aceitável.

A Tabela 1 mostra que, os valores obtidos da análise do método de Hargreaves (1976) apresentaram bons resultados para região e para os locais de estudo, por apresentarem coeficientes maiores que 0,88. Concordando com Silva et al.(2014), que o método se mostrou mais confiável e coerente, e também com Lacerda e Turco (2015), que verificaram os melhores ajustes na estimativa para a evapotranspiração diária, comparando ao Penman-Monteith.

Tabela 1. Valores do coeficiente de correlação (r), de concordância (d), de confiança (índice c), de determinação $\left(\mathrm{R}^{2}\right)$ e equação $(\mathrm{y}=\mathrm{ax})$ para o método de Hargreaves (1976).

\begin{tabular}{ccccccc}
$\begin{array}{l}\text { Hargreaves } \\
1976\end{array}$ & Equação & $\mathrm{R}^{2}$ & $\mathrm{~d}$ & $\mathrm{r}$ & $\begin{array}{c}\text { Índice } \\
\mathrm{C}\end{array}$ & Classificação \\
\hline Araxá & $\mathrm{y}=0,93 \mathrm{x}$ & 0,97 & 0,98 & 0,98 & 0,96 & Ótimo \\
$\begin{array}{c}\text { Campina Verde } \\
\text { Conceição das } \\
\text { Alagoas }\end{array}$ & $\mathrm{y}=0,88 \mathrm{x}$ & 0,97 & 0,95 & 0,98 & 0,94 & Ótimo \\
Ituiutaba & $\mathrm{y}=0,94 \mathrm{x}$ & 0,94 & 0,97 & 0,97 & 0,94 & Ótimo \\
Patrocínio & $\mathrm{y}=0,88 \mathrm{x}$ & 0,97 & 0,94 & 0,98 & 0,93 & Ótimo \\
Sacramento & $\mathrm{y}=0,89 \mathrm{x}$ & 0,99 & 0,96 & 0,99 & 0,95 & Ótimo \\
Uberlândia & $\mathrm{y}=0,92 \mathrm{x}$ & 0,97 & 0,97 & 0,98 & 0,96 & Ótimo \\
Regional & $\mathrm{y}=0,91 \mathrm{x}$ & 0,97 & 0,97 & 0,98 & 0,95 & Ótimo \\
& $\mathrm{y}=0,91 \mathrm{x}$ & 0,95 & 0,91 & 0,98 & 0,89 & Ótimo \\
\hline
\end{tabular}

Hargreaves (1976) apresentou o coeficiente de determinação acima de 0,94, mostrando-se adequado para a região, mas a sua necessidade de medição da radiação solar global dificulta sua utilização.
Os valores de $\mathrm{R}^{2}$ maiores que 0,94 indicam que o método apresentou precisão similar nos locais e regionalmente, quando comparados com o método PM. Mas ao adotar o coeficiente de determinação $\mathrm{R}^{2}$, como único 
critério de definição da qualidade de método para a região, não é adequado, uma vez que esse índice não estabelece o tipo e a magnitude das diferenças entre o valor estimado pelo métodopadrão e o valor previsto por modelos de estimativa (BARROS et al., 2009).

Observa-se com o índice de concordância "d" de Willmott, que o método de Hargreaves (1976) apresenta valores que mais se aproximam de uma concordância perfeita, próximos de 1 , entre 0,91 para avaliação regional e 0,98 para Araxá.

Para o coeficiente de confiança (índice c), o método de Hargreaves (1976) apresentou-se "Ótima” classificação em todas as localidades e também de forma regional, diferente da classificada "Boa” obtida por Silva et al. (2014), utilizando também o PM como padrão. Camargo e Sentelhas (1997), quando utilizaram como referência os lisímetros para o estado de São Paulo, encontraram a classificação "Ruim".

Os coeficientes de correlação apresentaram uma correlação muito forte positivamente na região e nos locais, sendo acima de 0,97 com o método Penman-Monteith FAO56, conforme a classificação de Camargo e Sentelhas (1997). Esses resultados do método superam os dados obtidos por de Silva et al. (2014), para a região de Fernando de Noronha, que foi de 0,78 .

A Tabela 2 apresenta os valores obtidos da análise de Hargreaves e Samani (1985) antes e após a calibração do HE, mostra que o método é adequado para a região, mas com a calibração houve uma melhora considerável dos coeficientes avaliados, aproximando mais de 1 .

Tabela 2 - Valores do expoente empírico (HE), do coeficiente de determinação $\left(\mathrm{R}^{2}\right)$, de correlação (r), de concordância (d) e de confiança (índice c) para o método de Hargreaves e Samani (1985), antes e após a calibração do HE.

\begin{tabular}{ccccccc}
$\begin{array}{c}\text { Hargreaves } \\
1985\end{array}$ & $\mathrm{HE}$ & $\mathrm{R}^{2}$ & $\mathrm{r}$ & $\mathrm{d}$ & $\begin{array}{r}\text { Índice } \\
\text { C }\end{array}$ & Classificação \\
\hline Araxá & 0,5 & 0,67 & 0,82 & 0,90 & 0,74 & Bom \\
Araxá* & 0,4998 & 0,70 & 0,84 & 0,91 & 0,76 & Muito bom \\
Campina Verde & 0,5 & 0,78 & 0,89 & 0,89 & 0,79 & Muito bom \\
$\begin{array}{c}\text { Campina Verde* } \\
\text { Conceição das } \\
\text { Alagoas }\end{array}$ & 0,4589 & 0,76 & 0,87 & 0,93 & 0,81 & Muito bom \\
$\begin{array}{c}\text { Conceição das } \\
\text { Alagoas* }\end{array}$ & 0,5 & 0,78 & 0,88 & 0,89 & 0,79 & Muito bom \\
Ituiutaba & 0,4582 & 0,82 & 0,90 & 0,94 & 0,85 & Ótimo \\
Ituiutaba* & 0,5 & 0,81 & 0,90 & 0,86 & 0,78 & Muito bom \\
Patrocínio & 0,4452 & 0,77 & 0,88 & 0,93 & 0,82 & Muito bom \\
Patrocínio* & 0,5 & 0,80 & 0,89 & 0,89 & 0,79 & Muito bom \\
Sacramento & 0,4579 & 0,77 & 0,88 & 0,94 & 0,82 & Muito bom \\
Sacramento* & 0,5 & 0,71 & 0,84 & 0,91 & 0,77 & Muito bom \\
Uberlândia & 0,4864 & 0,72 & 0,85 & 0,92 & 0,78 & Muito bom \\
Uberlândia* & 0,5 & 0,64 & 0,80 & 0,89 & 0,71 & Bom \\
Regional & 0,4918 & 0,65 & 0,81 & 0,89 & 0,72 & Bom \\
Regional* & 0,5 & 0,70 & 0,84 & 0,89 & 0,75 & Bom \\
*HE calibrado & 0,4712 & 0,70 & 0,84 & 0,92 & 0,77 & Muito bom \\
\hline and & & & & & &
\end{tabular}


Os coeficientes de correlação (r) apresentaram uma correlação muito forte, positivamente, em todas as situações para o método de Hargreaves e Samani (1985), sendo acima de 0,80, com o método Penman-Monteith FAO56. Concordando com Melo e Fernandes (2012) que encontram correlação (r) de 0,88 para Uberaba e também com Silva et al. (2011) que observaram 0,83 para Uberlândia em 2004.

O índice (d) menor foi de 0,86 , no entanto superior ao encontrado por Alencar et al.(2011) de 0,82 e Silva et al. (2011) de 0,84, na mesorregião.

O índice de concordância “d” de Willmott, que varia de 0 a 1 , representa o quanto os valores estimados se ajustam aos valores medidos, ou seja, é uma medida de quão bem o modelo estima o afastamento dos dados da média observada (WILLMOTT, 1985). Com os resultados obtidos, percebe-se que com a calibração, os valores de coeficiente de concordância foram maiores em relação à equação original, aumentando a aproximação de uma concordância perfeita.

As correlações " $\mathrm{R}$ "” obtidas entre os modelos Hargreaves (1985) e PM não foram afetadas diretamente pela calibração do método, por apresentar menores valores na localidade de Uberlândia com 0,64 e 0,65, maiores para Conceição das Alagoas com 0,82 e 0,78, e regionalmente manteve em 0,7 antes e após a calibração. Valores maiores foram encontrados por Carvalho et al.(2015) para Campina Verde $(0,86)$ e para Uberaba $(0,89)$. Isto ocorreu provavelmente devido o modelo ter sido desenvolvido em condições de clima árido (HARGREAVES; SAMANI, 1985).

Os valores obtidos da análise do método de Hargreaves (1994) antes e após a calibração do HE são apresentados na Tabela 3, indicando que o método é adequado para os locais e para a região. Com a calibração houve uma melhora nos coeficientes.

O valor do expoente empírico HE, para região foi de 0,4598 e para localidades apresentaram entre 0,4259 (Patrocínio) e 0,4907 (Araxá), mantendo-se abaixo do original e diferente de 0,5. Coincidindo com Kelso-Bucio et al. (2012) quando calibrou para 32 estações no México, com Trajkovi (2007) no Sudeste da Europa e com Conceição (2013) no Noroeste Paulista. Mas Fernandes et al. (2012) averiguou valores de expoentes maiores que 0,59 para o estado de Goiás.

Tabela 3. Valores do expoente empírico (HE), do coeficiente de correlação (r), de concordância (d), de confiança (índice c) e de determinação $\left(\mathrm{R}^{2}\right)$ para o método de Hargreaves (1994) antes e após a calibração do HE.

\begin{tabular}{|c|c|c|c|c|c|c|}
\hline $\begin{array}{l}\text { Hargreaves } \\
1994\end{array}$ & $\mathrm{HE}$ & $\mathrm{R}^{2}$ & $\mathrm{r}$ & d & $\begin{array}{l}\text { Índice } \\
\text { C }\end{array}$ & Classificação \\
\hline Araxá & 0,5 & 0,65 & 0,80 & 0,89 & 0,72 & Bom \\
\hline Araxá* & 0,4907 & 0,67 & 0,82 & 0,90 & 0,74 & Bom \\
\hline Campina Verde & 0,5 & 0,76 & 0,87 & 0,87 & 0,76 & Bom \\
\hline Campina Verde* & 0,4503 & 0,74 & 0,86 & 0,92 & 0,79 & Muito bom \\
\hline $\begin{array}{l}\text { Conceição das } \\
\text { Alagoas }\end{array}$ & 0,5 & 0,77 & 0,88 & 0,88 & 0,77 & Muito bom \\
\hline $\begin{array}{l}\text { Conceição das } \\
\text { Alagoas* }\end{array}$ & 0,4505 & 0,80 & 0,89 & 0,94 & 0,84 & Muito bom \\
\hline Ituiutaba & 0,5 & 0,80 & 0,89 & 0,83 & 0,75 & Bom \\
\hline Ituiutaba* & 0,4382 & 0,76 & 0,87 & 0,93 & 0,81 & Muito bom \\
\hline Patrocínio & 0,5 & 0,78 & 0,89 & 0,86 & 0,76 & Muito bom \\
\hline Patrocínio* & 0,4259 & 0,75 & 0,87 & 0,90 & 0,78 & Muito bom \\
\hline
\end{tabular}


Turco et al.

\begin{tabular}{|c|c|c|c|c|c|c|}
\hline Sacramento & 0,5 & 0,70 & 0,83 & 0,90 & 0,75 & Bom \\
\hline Sacramento* & 0,4773 & 0,70 & 0,84 & 0,91 & 0,76 & Muito bom \\
\hline Uberlândia & 0,5 & 0,61 & 0,78 & 0,88 & 0,69 & Bom \\
\hline Uberlândia* & 0,4854 & 0,63 & 0,79 & 0,88 & 0,70 & Bom \\
\hline Regional & 0,5 & 0,69 & 0,83 & 0,87 & 0,72 & Bom \\
\hline Regional $^{*}$ & 0,4598 & 0,68 & 0,83 & 0,91 & 0,76 & Bom \\
\hline
\end{tabular}

*HE calibrado.

A correlação (r) entre Hargreaves (1994) e o de PM apresentou-se muito forte positivamente com valores acima de 0,78. Coincidindo com Conceição (2013) que encontrou valores acima de 0,88 antes e após a calibração.

O índice de concordância apresentou valores próximos aos valores de Conceição (2013) e houve aumento de valores após calibração na região, tendendo para uma concordância perfeita.

A classificação do método quanto ao índice de confiança "c" apresentou-se bom ou muito bom, entre 0,691 (Uberlândia, HE 0,5) e 0,838 (Conceição das Alagoas). Mas sempre evoluindo acima de 0,018 com a calibração local e na regional o aumento foi de 0,032 mantendose como bom, justificando o ajuste do expoente empírico para o método, mas apresentando inferior a evolução que Conceição (2013) encontrou com a calibração do HE de 0,11, passando de bom $(0,69)$ para muito bom $(0,81)$.

Os coeficientes de determinação " $\mathrm{R}$ "” não foram afetados pela calibração do método por apresentar valores menores ou iguais a equação original (HE 0,5). Coincidindo com Conceição et al. (2013) que o $\mathrm{R}^{2}$ diminuiu de 0,81 para 0,79 e oposto ao observado por Fernandes et al. (2012) aumentando de 0,49 para 0,55, confirmando assim que tal coeficiente não está diretamente ligado a calibração.

A análise de regressão no modelo linear sem intercepto da ETo, determinada pelo método Hargreaves (1976), Figura 2, em comparação com o método PM, apresentou valores que superestimaram os valores médios diários da ETo, sendo oposto ao encontrado por Lacerda e Turco (2015), que avaliaram o mesmo método para o município de Uberlândia em 2010. A superestimação de Hargreaves 1976 em relação a PM deve ser devido a interferência dos parâmetros de umidade e vento na evapotranspiração de referência de toda a região.

Visualiza-se, na Figura 2 a análise de regressão linear $(\mathrm{y}=\mathrm{a} \mathrm{x}+\mathrm{b})$ entre o Hargreaves e Samani (1985) e o PM antes e após a calibração para a região, com suas respectivas equações de ajuste e coeficiente de determinação $\left(\mathrm{R}^{2}\right)$. O método de Hargreaves (1985) superestima o padrão. No estado do Ceará, Lima Junior et al. (2016) comprovou que Hargreaves (1985) independente de calibrado ou não, se apresenta superestimado em relação ao PM. A superestimação conduz ao aumento na lâmina de água a ser aplicada e como consequência interfere na umidade ideal do solo para a absorção adequada pela planta de solução presente no solo.

A Figura 2 exibe a análise de regressão linear $(\mathrm{y}=\mathrm{a} \mathrm{x}+\mathrm{b})$, pelo método de Hargreaves (1994), que superestima o padrão. Kelso-Bucio et al. (2012) afirmam que a precisão aumenta com a calibração da equação, podendo ser percebida observando-se a proximidade dos pontos a reta. 


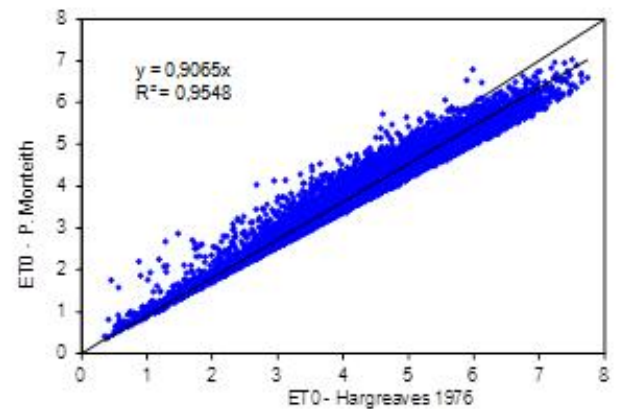

Antes da calibração Regional, anos impares

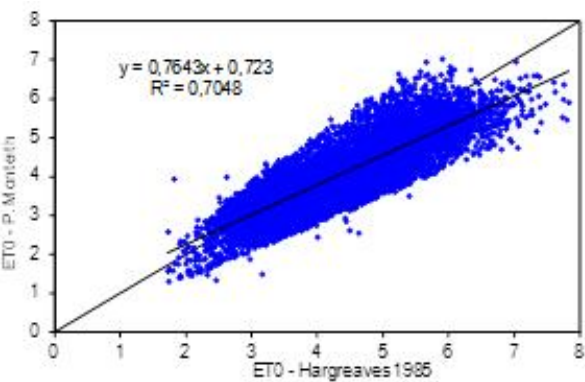

Antes da calibração Regional, anos impares

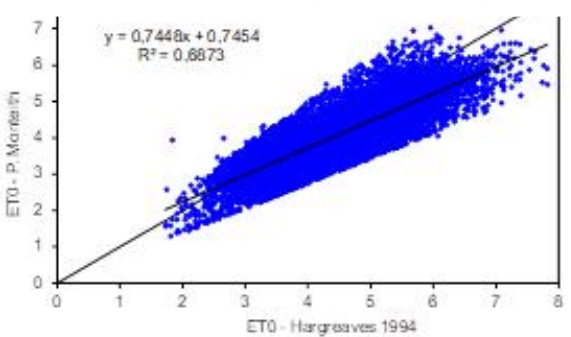

Depois da Calibração Regional, anos pares

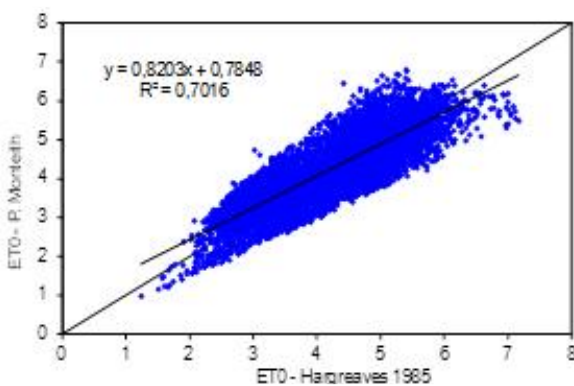

Depois da Calibração Regional, anos pares

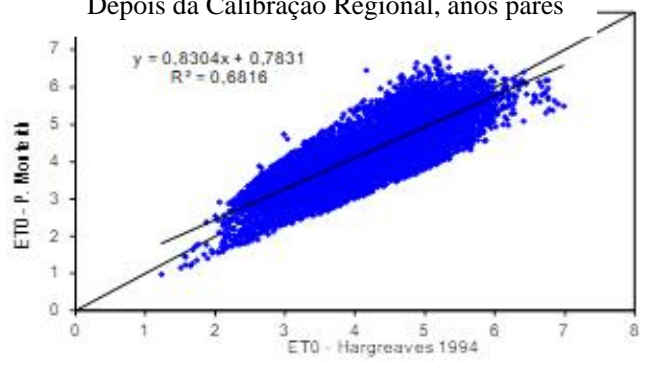

Figura 2. Correlação entre a os valores diários da ETo, obtidos com as equações de Hargreaves, em relação ao Penman-Monteith, para a região do Alto Paranaíba e Triângulo Mineiro. Sendo: equação de Hargreaves (1976); de Hargreaves e Samani (1985); Hargreaves (1994).

Na Tabela 4 pode-se observar os resultados obtidos, utilizando-se os critérios estatísticos, para quantificar o desempenho das equações EToRS e EToRG, ajustadas para o processo local e regional. No método de
Hargreaves (1985), a eficiência do modelo variou de 0,62 (Uberlândia) e a 0,77 (Conceição das Alagoas). As estações Araxá e Uberlândia apresentaram os maiores valores de ERQM e EMA para os dois métodos.

Tabela 4 . Desempenho das equações EToHSR e EToHGR ajustadas para o processo local e regional.

$\begin{array}{ll} & \text { Hargreaves (1994) Hargreaves (1985) }\end{array}$

$\begin{array}{lllll}\text { EF } & \text { ERQM \% } & \text { EMA } & \text { EF } & \text { ERQM \% }\end{array}$

\begin{tabular}{cllllll}
\hline Araxá & 0,66 & 14,93 & 0,54 & 0,69 & 14,20 & 0,51 \\
Campina Verde & 0,70 & 12,14 & 0,43 & 0,73 & 11,61 & 0,41 \\
Conceição das & 0,76 & 11,39 & 0,38 & 0,77 & 11,04 & 0,37 \\
Alagoas & 0,73 & 11,40 & 0,39 & 0,75 & 11,05 & 0,37 \\
Ituiutaba & 13,59 & 0,46 & 0,74 & 11,55 & 0,38 \\
Patrocínio & 0,64 & 13,27 & 0,47 & 0,69 & 12,87 & 0,45 \\
Sacramento & 0,67 & 14,88 & 0,53 & 0,62 & 14,29 & 0,51 \\
Uberlândia & 0,59 & 13,14 & 0,46 & 0,71 & 12,45 & 0,43 \\
Regional & 0,68 & & & & \\
\hline
\end{tabular}

EF - eficiência do modelo; ERQM - erro relativo do quadrado médio; EMA - erro médio absoluto. 
A calibração regional apresentou melhor desempenho no método de Hargreaves e Samani(1985) em relação a Hargreaves (1994). Provavelmente, devido ao método de Hargreaves (1985) ter a temperatura média diária do ar, ocasionando maior precisão.

\section{CONCLUSÃO}

As equações de Hargreaves são uma alternativa eficiente para estimar a ETo diária em locais do Triângulo Mineiro e Alto Paranaíba, em que a disponibilidade de dados climáticos é limitada.

\section{REFERÊNCIAS}

ALENCAR, L. P.; DELGADO, R. C.; ALMEIDA, T. S.; WANDERLEY, H. S. Comparação de diferentes métodos de estimativa diária da evapotranspiração de referência para a região de Uberaba. Revista Brasileira de Ciências Agrárias, v.6, p.337343, 2011.

ALLEN, R. G. Assessing integrity of weather data for reference evapotranspiration estimation. Journal of Irrigation and Drainage Engineering, v. 122, p. 97-106, 1996. doi: http://dx.doi.org/10.1061/(ASCE)07339437(1996)122:2(97).

ALLEN, R. G.; PEREIRA, L. S.; RAES, D.; SMITH, M. Evapotraspiration del cultivo: guias para la determinación de los requerimientos de água de los cultivos. Roma: FAO, 2006. 298 p. (Estúdio Riego e Drenaje, Paper 56).

BARROS, V. R.; SOUZA, A.P.; FONSECA, D.C.; SILVA, L. B. D. Avaliação da evapotranspiração de referência na Região de Seropédica, Rio de Janeiro, utilizando lisímetro de pesagem e modelos matemáticos. Revista Brasileira de Ciências Agrárias, v. 4, p. 198203, 2009.
BERTI, A.; TARDIVO, G.; CHIAUDANI, A.; RECH, F.; BORIN, N. Assessing reference evapotranspiration by the Hargreaves method in north-eastern Italy. Agricultural Water Management. v.140, p.20-25, 2014. doi: https://doi.org/10.1016/j.agwat.2014.03.015

CAMARGO, A. P.; SENTELHAS, P. C. Avaliação do desempenho de diferentes métodos de estimativa da evapotranspiracão potencial no Estado de São Paulo, Brasil. Revista Brasileira de Agrometeorologia, v. 5, p. 89-97, 1997.

CARVALHO, D. F. DE; ROCHA, H. S. DA; BONOMO, R.; SOUZA, A. P. de Estimativa da evapotranspiração de referência a partir de dados meteorológicos limitados. Pesquisa Agropecuária Brasileira, v.50, n.1, p.1-11, 2015. doi: http://dx.doi.org/10.1590/S0100204X2015000100001

CERVANTES-OSORNIO, R.; ARTEAGARAMÍRES, R.; VÁZQUEZ-PEÑA, M.A.; OJEDA-BUSTAMANTE, W.; QUEVEDONOLASCO, A. Modelos Hargreaves PriestleyTaylor y redes neuronales artificiales en la estimación de la evapotranspiración de referencia. Ingenieria Investigación y Tecnologia. v. XIV, n. 2, p. 163-176, 2013.

CONCEIÇÃO, M. A. F. Ajuste do modelo de Hargreaves para estimativa da evapotranspiração de referência no noroeste paulista. Revista Brasileira de Agricultura Irrigada, v.7, n.5, p. 306-316, 2013. doi: 10.7127/RBAI.V7N500172

FENG, Y.; JIA, Y.; CUI, N.; ZHAO, L.; LI, C.; GONG, D. Calibration of Hargreaves model for reference evapotranspiration estimation in Sichuan basin of southwest China. Agricultural Water Management. v.181, p.1-9, 2017. doi: https://doi.org/10.1016/j.agwat.2016.11.010

FERNANDES, D. S.; HEINEMANN, A. B.; PAZ, R. L. F.; AMORIM, A. O. Calibração regional e local da equação de Hargreaves para estimativa da evapotranspiração de referência. 
Revista Ciência Agronômica, v.43, n.2, p. 246-255, 2012.

HARGREAVES, G.H. Climate and irrigation requirements for Brazil. Logan, Utah State Universitey, 1976. 44 p.

HARGREAVES, G.H. Defining and using reference evapotranspiration. Journal of Irrigation and Drainage Engineering, v.120, n.6, p.1132-1139, 1994.

HARGREAVES, G. H.; SAMANI, Z. A. Reference crop evapotranspiration from temperature. Applied Engineering in Agriculture, v. 01, p. 96-99, 1985.

HEYDARI, M.M; HEYDARI, M. "Calibration of Hargreaves-Samani equation for estimating reference evapotranspiration in semiarid and arid regions”. Archives of Agronomy and Soil Science, v. 60, p. 695-713, 2014 . doi: https://doi.org/10.1080/03650340.2013.808740

KELSO-BUCIO, H.A.; BÂ, K.M.; SÁNCHEZMORALES, S.; REYES-LÓPEZ, D. Calibración del exponente de la ecuación Hargreaves-ETo en los estados de Chiapas, Oaxaca, Puebla y Veracruz, México. Agrociencia, v.46, n.3, p.221-229, 2012.

LACERDA, Z. C.; TURCO, J. E. P. Estimation methods of reference evapotranspiration (ETo) for Uberlândia - MG. Engenharia Agrícola, v.35, n.1, p. 27- 38, 2015. doi: http://dx.doi.org/10.1590/1809-4430-

Eng.Agric.v35n1p27-38/2015

LIMA JUNIOR, J. C.; ARRAES, F. D. D.; OLIVEIRA, J. B.; NASCIMENTO, F. A. L.; MACÊDO, K. G. Parametrização da equação de Hargreaves e Samani para estimative da evapotranspiração de referência no Estado do Ceará, Brasil. Revista Ciência Agronômica, v.47, n.3, p.447-454, 2016. doi: 10.5935/18066690.20160054

MELO, G. L. de; FERNANDES, A. L. T. Evaluation of empirical methods to estimate reference evapotranspiration in Uberaba, State of Minas Gerais, Brazil. Engenharia Agrícola, Jaboticabal, v.32, n.5, p.875-888. 2012.

MEHDIZADEH, S.; SAADATNEJADGHARAHASSANLOU, H.; BEHMANESH, J. "Calibration of Hargreaves-Samani and Priestley - Taylor equations in estimating reference evapotranspiration in the Northwest of Iran”. Archives of Agronomy and Soil Science, v.63, p. 1-14, 2016. doi: https://doi.org/10.1080/03650340.2016.

MOURA, A.R.C.; MONTENEGRO, S.M.G.L.; ANTONINO, A.C.D.; AZEVEDO, J.R.G.; SILVA, B.B.; Oliveira, L.M.M. Evapotranspiração de referência baseada em métodos empíricos em bacia experimental no estado de Pernambuco - Brasil. Revista Brasileira de Meteorologia, v.28, n.2, p.181-191. 2013.

PILAU, F.G.; BATTISTI, R.; SOMAVILLA, L.; RIGHI, E.Z. Desempenho de métodos de estimativa da evapotranspiração de referência nas localidades de Frederico Westphalen e Palmeira das Missões - RS. Ciência Rural, Santa Maria, v.42, n.2, p.283-290, 2012.

RAMOS, F.; OCCHIPINTI, A. G.; VILA NOVA, N. A.; REICHART, K.; MAGALHÃES, P. C.; CLEARY, R. W. Engenharia hidrológica. Rio de Janeiro: UFRJ, 1989. 404p.

RAZIEI, T.; PEREIRA, L.S. Estimation of ETo Hargreaves - Samani and FAO-PM temperature methods for a wide range of climates in Iran. Agricultural Water Management. v.121, p.118, 2013.2 doi: https://doi.org/10.1016/j.agwat.2012.12.019

SILVA, J. R. L.; MONTENEGRO, A. A. A.; SANTOS, T. E. M.; SANTOS, E. S. Desempenho de diferentes métodos de estimativa da evapotranspiração de referência para Fernando de Noronha. Irriga, v.19, n.3, p. 390-404, 2014.

SILVA, V. J.; CARVALHO, H. P.; DA SILVA, C. R.; CAMARGO, R.; TEODORO, R. E. F. Desempenho de diferentes métodos de 
estimativa da evapotranspiração de referência diária em Uberlândia-MG. Bioscience Journal, v. 27, n.1, p. 95-101, 2011.

TRAJKOVIC, S. Hargreaves versus PenmanMonteith under humid conditions. Journal of Irrigation and Drainage Engineering. v.133, n.1, p. 38-42. 2007.
WILLMOTT, C.J. On the validation of models. Physical Geography, v.2, p.184-194, 1985.

WRAITH, J. M.; OR, D. Nonlinear parameter estimation using spreadsheet software. Journal of Natural Resources and Life Sciences Education, v. 27, p. 13-19, 1998. 